\title{
SYLVICULTURE DU HÊTRE : CONTRAINTES, ENJEUX, ORIENTATIONS DE GESTION
}

\author{
Yves Bastien - Sebastian Hein - Alain Chavane
}

Deuxième essence feuillue après le Chêne sessile et pédonculé, le Hêtre représente en France, avec une surface totale de près de 1300000 ha, un enjeu économique important. D'après les données de l'Inventaire forestier national (IFN) de 1999, avant les tempêtes de décembre, les structures de futaie étaient largement représentées avec $65 \%$ de la surface totale des peuplements de Hêtre prépondérant, contre $26 \%$ pour les mélanges futaie-taillis, en évolution progressive vers la futaie du fait de l'abandon du traitement de taillis-sous-futaie.

Le travail qui suit porte sur les peuplements de futaie régulière et les mélanges futaie-taillis qui constituent à eux deux l'ossature des hêtraies françaises. Nous identifierons d'abord les contraintes et les enjeux en présence pour proposer ensuite des orientations de gestion qui porteront essentiellement sur les futaies régulières de Hêtre en croissance, peuplements pour lesquels les objectifs d'amélioration nous semblent prioritaires. La gestion des mélanges futaietaillis, qui s'inscrit dans un cadre de gestion plus ouvert avec les traitements irréguliers, sera illustrée par un exemple abordé à la fin de ce travail.

\section{CONTRAINTES}

\section{Des futaies régulières denses et pures majoritaires, et à risques}

Les futaies régulières de Hêtre sont, pour une part importante, constituées de peuplements denses et purs, sensibles aux dégâts de vent et à certains parasites (chancre en particulier), et producteurs de bois souvent colorés et nerveux. L'importance de l'enjeu de la gestion qualitative de la hêtraie régulière se mesure aujourd'hui à l'aune des problèmes rencontrés, accentués par les dégâts considérables de la tempête de 1999 qui ont complètement déséquilibré la hêtraie - lorraine en particulier -, la privant d'une très grande part de ses gros bois et mettant par conséquent à mal la continuité de sa production.

Ces peuplements présentent des risques accrus dans un environnement changeant au regard de divers facteurs :

- dégâts de vent avec une forte sensibilité pour tous les peuplements qui dépassent 23 mètres de hauteur dominante (Vinkler, 2002) ;

- forte augmentation de la productivité calculée sur un siècle pour des peuplements d'âge inférieur à 80 ans : 90 à 120 \% sur la largeur de cernes (Dhôte et al., 2000), plus de 50 \% sur la vitesse de croissance en hauteur (Bontemps, 2003) ;

- risque accru de chablis avec l'augmentation de la hauteur des jeunes peuplements, constatée ci-avant ;

- réchauffement climatique, risque de dysfonctionnement de la hêtraie. 
Yves Bastien - Sebastian Hein - Alain Chavane

\section{Des peuplements hérités du taillis-sous-futaie, très diversifiés}

Les mélanges futaie-taillis, au sens de l'IFN, qui sont des peuplements hérités du taillis-sousfutaie orientés dans des processus de conversion en futaie, recouvrent diverses situations. Si, dans le passé, les schémas de conversion en futaie régulière ont été longtemps la règle en forêt publique, les itinéraires sylvicoles actuels semblent plutôt privilégier des voies de conversion douce en futaie irrégulière lorsque la variabilité des conditions de station accentue la variabilité originelle des peuplements. Cette orientation récente s'appuie sur la nécessité d'optimiser la production, en tirant parti du mieux possible des potentialités des essences en place, par une sylviculture d'arbres non normative, soucieuse d'améliorer l'existant tout en limitant les dépenses. Cette nouvelle sylviculture reste à formaliser.

\section{Une faible proportion de bois de qualité}

Le bois de Hêtre a des défauts connus liés pour une part à sa sylviculture : accroissements fins générant des bois nerveux, cœur rouge ${ }^{(1)}$, nœuds recouverts - souvent noirs après élagage tardif de grosses branches -, cannelure.

Ces défauts pénalisent lourdement la qualité du bois, et la production des hêtraies génère une faible proportion de bois de haute qualité ainsi qu'en témoigne le tableau I (ci-dessous).

tABleau I Volumes commercialisés de lots purs de Hêtre "faconnés bord de route" par qualité et prix moyens

Les qualités A, B, C et D correspondent aux normes de classement du CTBA. La qualité A exclut les bois dont les cernes annuels sont inférieurs à $4 \mathrm{~mm}$.

\begin{tabular}{|c|c|c|c|c|c|}
\hline \multirow{2}{*}{ Qualité } & \multicolumn{3}{|c|}{$\begin{array}{c}\text { Lorraine } \\
(1994-1996)\end{array}$} & \multicolumn{2}{|c|}{$\begin{array}{c}\text { Bade-Wurtemberg } \\
\quad(1978-1999)\end{array}$} \\
\hline & $\begin{array}{c}137000 \mathrm{~m}^{3} / \mathrm{an} \\
\mathrm{D} \mathrm{exclu}\end{array}$ & $\begin{array}{l}212600 \mathrm{~m}^{3} / \mathrm{an} \\
\text { totalité }\end{array}$ & $\operatorname{Prix} € / \mathrm{m}^{3}$ & $114300 \mathrm{~m}^{3} / \mathrm{an}$ & $\operatorname{Prix} € / \mathrm{m}^{3}$ \\
\hline$A \ldots \ldots \ldots \ldots \ldots$ & $1 \%$ & $0,6 \%$ & 322 & $1 \%$ & 266 \\
\hline$B \ldots \ldots \ldots \ldots \ldots$ & $24 \%$ & $15,8 \%$ & 168 & $44 \%$ & 97 \\
\hline$c \ldots \ldots \ldots \ldots \ldots \ldots$ & $75 \%$ & $48,1 \%$ & 81 & $55 \%$ & 41 \\
\hline$D \ldots \ldots \ldots \ldots \ldots \ldots$ & & $35,5 \%$ & 38 & & \\
\hline
\end{tabular}

Sources : ONF Lorraine et données allemandes (Ministerium für Ländlichen Raum, Ernährung Landwirtschaft und Forsten BadenWürttemberg).

La comparaison des qualités et des prix entre la Lorraine et le Bade-Wurtemberg doit être faite avec une certaine prudence, compte tenu des pratiques différentes de classement des bois qui y sont mises en œuvre. On peut toutefois dégager de ce tableau les tendances suivantes :

- la production de qualité $A$ est très faible, moins de $1 \%$ du volume grume total commercialisé si on intègre la qualité $D$ (exclue en Allemagne et transformée en bois de trituration ou de chauffage) ;

- pour la Lorraine, la belle qualité globale $(A+B)$ ne représente que 16,4 \% du total commercialisé et les prix au $\mathrm{m}^{3}$ sont divisés par deux à chaque fois que l'on baisse d'une classe de qualité en partant de la classe supérieure ;

(1) Le cœur rouge peut être lié à l'âge des arbres ou à la présence de fourches ou branches cassées dans la partie supérieure du fût. Une hêtraie dense, constituée d'arbres à faibles accroissements individuels, a un cycle de production long (supérieur à 120 ans) et produit de nombreux arbres à cœur rouge. 
Photo 1 - Cœur rouge (ci-contre)

Pour qu'un Hêtre soit classable en qualité A, il faut que le cœur rouge soit contenu dans un noyau central ne dépassant pas le tiers du diamètre de l'arbre. Des sous-classes "A et B rouges" autorisant $100 \%$ de cœur rouge sain et homogène peuvent être admises (norme AFNOR 1997).

Photos Y. BASTIEN

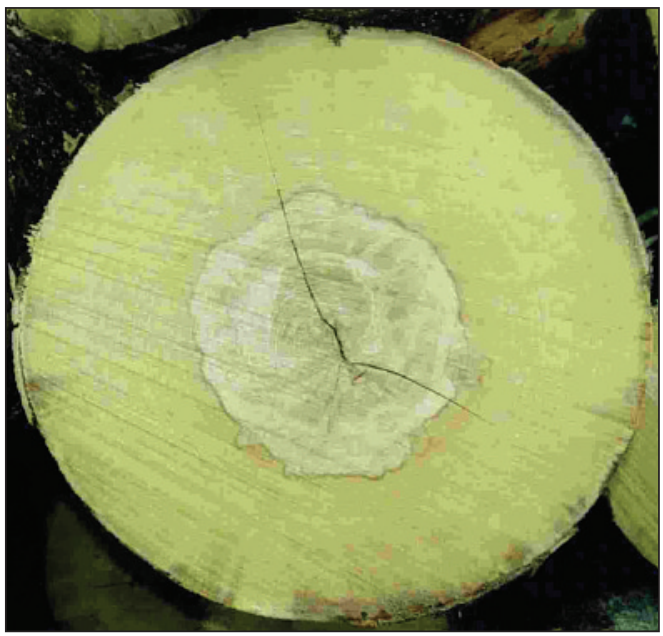

Photo 2 - Nœud noir (ci-dessous, à gauche)

Les nœuds noirs, qui surviennent après élagage naturel tardif des grosses branches situées à la base du houppier, peuvent être évités par des éclaircies fortes par le haut entretenant un bon éclairement de la partie inférieure des couronnes des arbres. Les branches obliques, à l'origine de plaie d'élagage à cicatrisation lente, génèrent des cavités fréquentes qui altèrent les billes de pied.

Photo 3 - Cannelure (ci-dessous, à droite)

Prenant naissance sous le point d'insertion d'une branche ombragée, la cannelure, défaut rédhibitoire, peut affecter toute la bille de pied et la déclasser totalement.
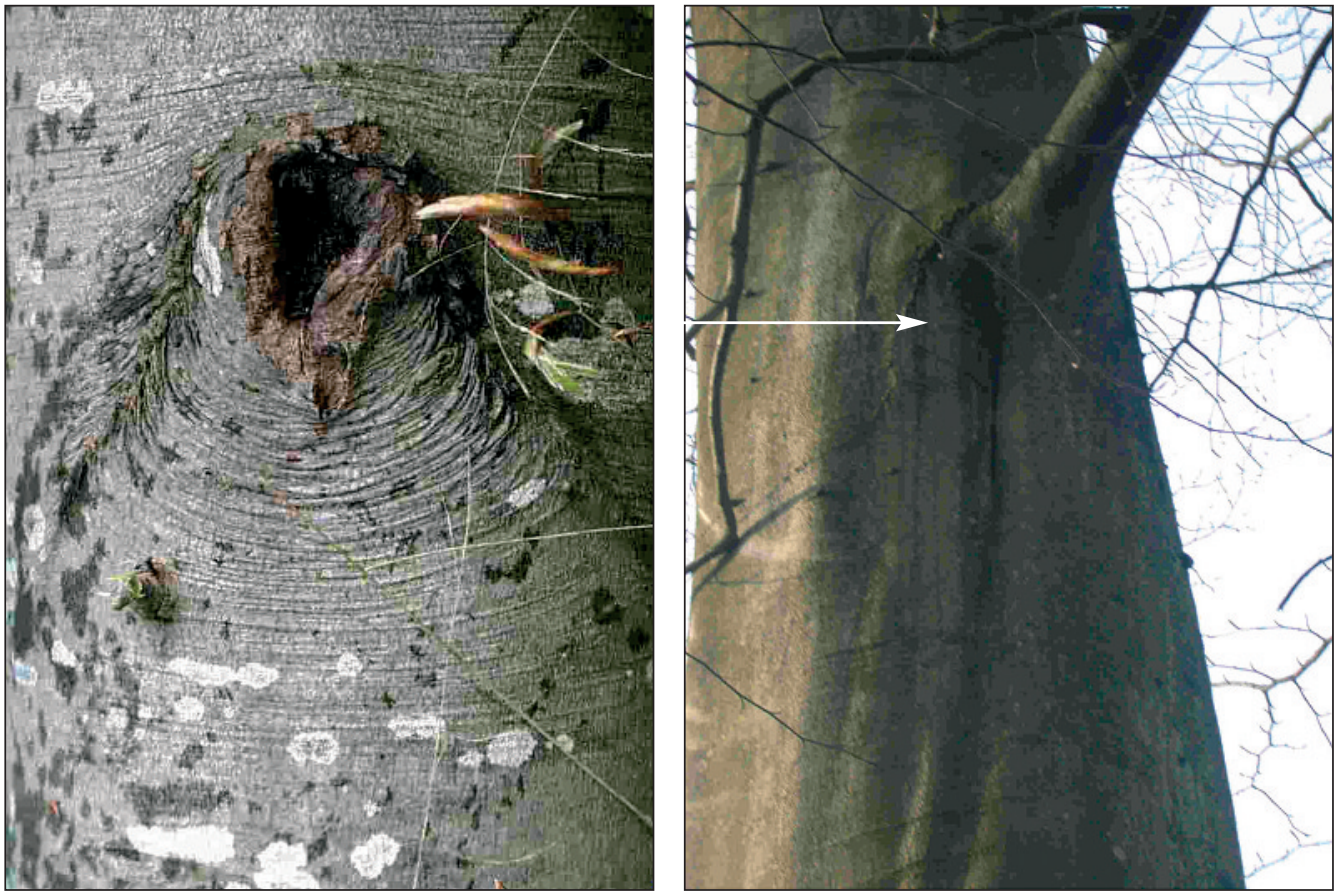
Yves Bastien - Sebastian Hein - Alain Chavane

- les écarts de prix constatés entre la Lorraine et l'Allemagne peuvent s'expliquer, outre la remarque préalable faite ci-avant, par le fait que les grumes lorraines, issues plutôt des anciens taillis-sous-futaie de forêts communales, présentent de larges cernes, alors que les bois de futaie à faibles accroissements sont prépondérants en Allemagne.

La sylviculture dispose donc d'une certaine marge de manœuvre pour améliorer la production qualitative des hêtraies.

\section{ENJEUX}

\section{Mieux gérer les risques}

La gestion des risques accrus, et des dégâts de vent en particulier, s'appuie sur deux grands axes :

- l'amélioration du fonctionnement de l'écosystème hêtraie en privilégiant le mélange d'essences diverses d'autécologie différente est mieux à même de favoriser sa résilience ${ }^{(2)}$ par la dynamique de la régénération naturelle initiée par le mélange ;

- des objectifs sylvicoles visant à réduire les risques : abaissement des âges d'exploitabilité et décapitalisation progressive conjuguent leurs effets pour limiter le développement en hauteur des arbres et les pertes économiques éventuelles (moins de volume sur pied), et encourager les dynamiques forestières naturelles et donc la résilience des peuplements (mélanges d'essences, régénération naturelle préexistante).

\section{Améliorer la production qualitative en limitant les coûts}

Cet objectif vise à :

- produire rapidement le maximum de gros bois de qualité $A$ et $B$ dans les futaies pour combler le déficit actuel de cette catégorie de produits ;

- réussir la conversion des anciens taillis-sous-futaie en adaptant les itinéraires sylvicoles à la variabilité des peuplements.

Il implique la mise en œuvre d'une gestion à coûts gradués en fonction des potentialités des stations forestières dans le souci d'une recherche d'économie de moyens.

Ces considérations nous amènent donc à limiter ces objectifs d'optimisation de la gestion qualitative de la hêtraie dans les peuplements situés en station de productivité suffisante (au moins 5 à $6 \mathrm{~m} 3 / \mathrm{ha} / \mathrm{an}$ d'accroissement moyen) et en conditions normales de relief, c'est-à-dire sans pente excessive susceptible d'entraîner des surcoûts d'exploitation.

\section{Un enjeu central de forte intensification des prélèvements en éclaircie dans tous les peuplements productifs de futaie}

Cet enjeu, qui rassemble tous les objectifs définis précédemment, revêt une importance grandissante dans un contexte perturbé :

- augmentation de productivité et tendance naturelle des hêtraies à accumuler un trop fort capital sur pied par suite de trop faibles prélèvements, notamment dans les jeunes peuplements ;

- forêts mutilées et appauvries en gros bois ;

- forestiers psychologiquement éprouvés par les tempêtes et plus enclins à la prudence.

(2) La résilience d'un écosystème forestier caractérise sa capacité naturelle à se régénérer naturellement en cas de dommages. 
Il en découle des orientations de gestion plutôt centrées, comme nous l'avons dit précédemment, sur les peuplements en croissance de futaie régulière.

\section{ORIENTATIONS DE GESTION}

\section{En futaie régulière}

- Quel matériel sur pied?

Intérêt des faibles matériels sur pied pour stimuler une forte croissance en diamètre

La synthèse des résultats des places d'expérience sur les éclaircies de Hêtre en France : Darney (Vosges), La Haye d'Aubenton (Aisne), Haye-Charlemagne (Meurthe-et-Moselle), Jeandelaincourt (Meurthe-et-Moselle), La Verrière du Grosbois (Doubs) et à l'étranger : Totterup (Danemark), montre qu'une largeur moyenne de cernes de $4 \mathrm{~mm}$ minimum, critère de classement des bois en qualité A (norme AFNOR, 1997), ne peut être obtenue qu'avec des surfaces terrières inférieures à $20 \mathrm{~m}^{2} /$ ha après éclaircie dans des peuplements âgés de 26 à 134 ans en bonne station (figure 1, ci-dessous).

D’autres résultats, obtenus par simulation avec CAPSIS (Seynave, 1999 ; Comtesse, 2000), préconisent des interventions très fortes dans le jeune âge pour stimuler la croissance en diamètre en s'appuyant sur des itinéraires basés sur des surfaces terrières après éclaircie inférieures à $15 \mathrm{~m}^{2} /$ ha jusqu'à 50 ans et inférieures à $20 \mathrm{~m}^{2}$, au-delà.

\section{FIGURE 1 LIAISON LARGEUR (LC) DE CERNES/SURFACE TERRIÈRE APRÈS ÉCLAIRCIE (G)}

Les largeurs moyennes de cernes sont calculées à partir des diamètres quadratiques moyens (Dg) ou des diamètres dominants (Do) et par différence sur les diamètres correspondants en début et fin de périodes de croissance indiquées entre parenthèses :

- Dg peuplement pour Darney (60-77 ans), Totterup (19-68 ans),

- Dg arbres-objectifs : 400 arbres pour Haye d'Aubenton (16-26 ans), 66 arbres pour Jeandelaincourt (1-77 ans), 80 à 100 arbres pour La Verrière du Gros Bois (30-35 ans),

- Do pour Haye-Charlemagne (49-134 ans).

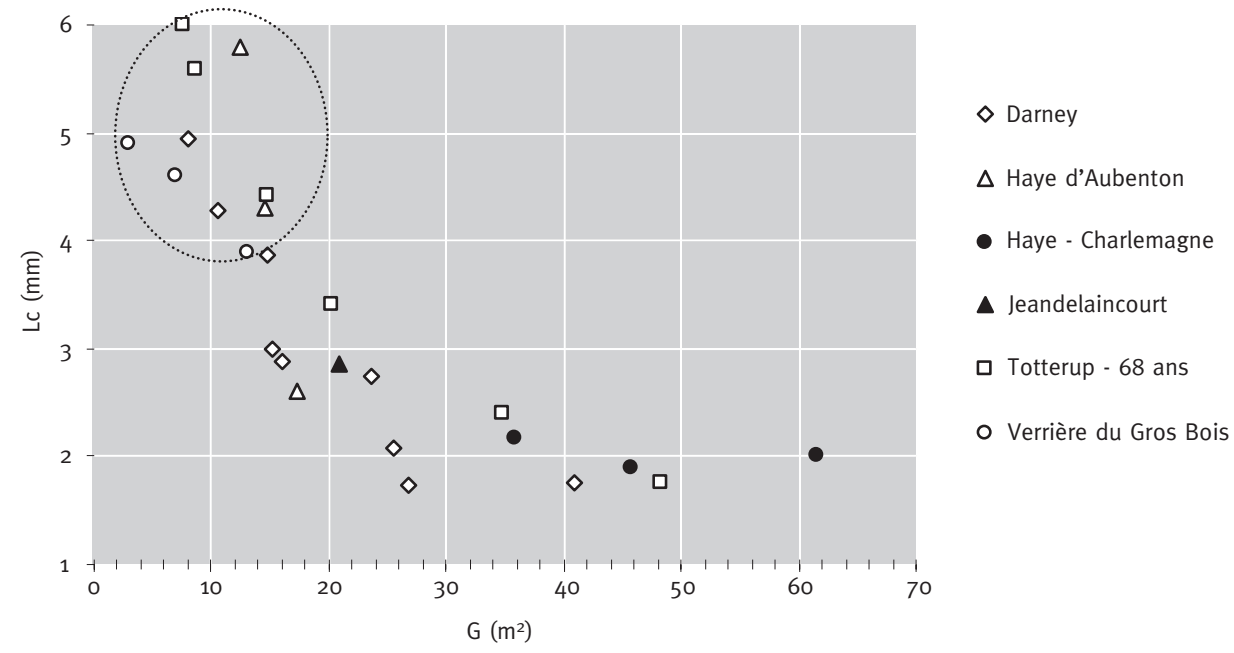


Yves Bastien - Sebastian Hein - Alain Chavane

Des Hêtres à houppiers très développés

pour produire le plus vite possible des gros bois de qualité

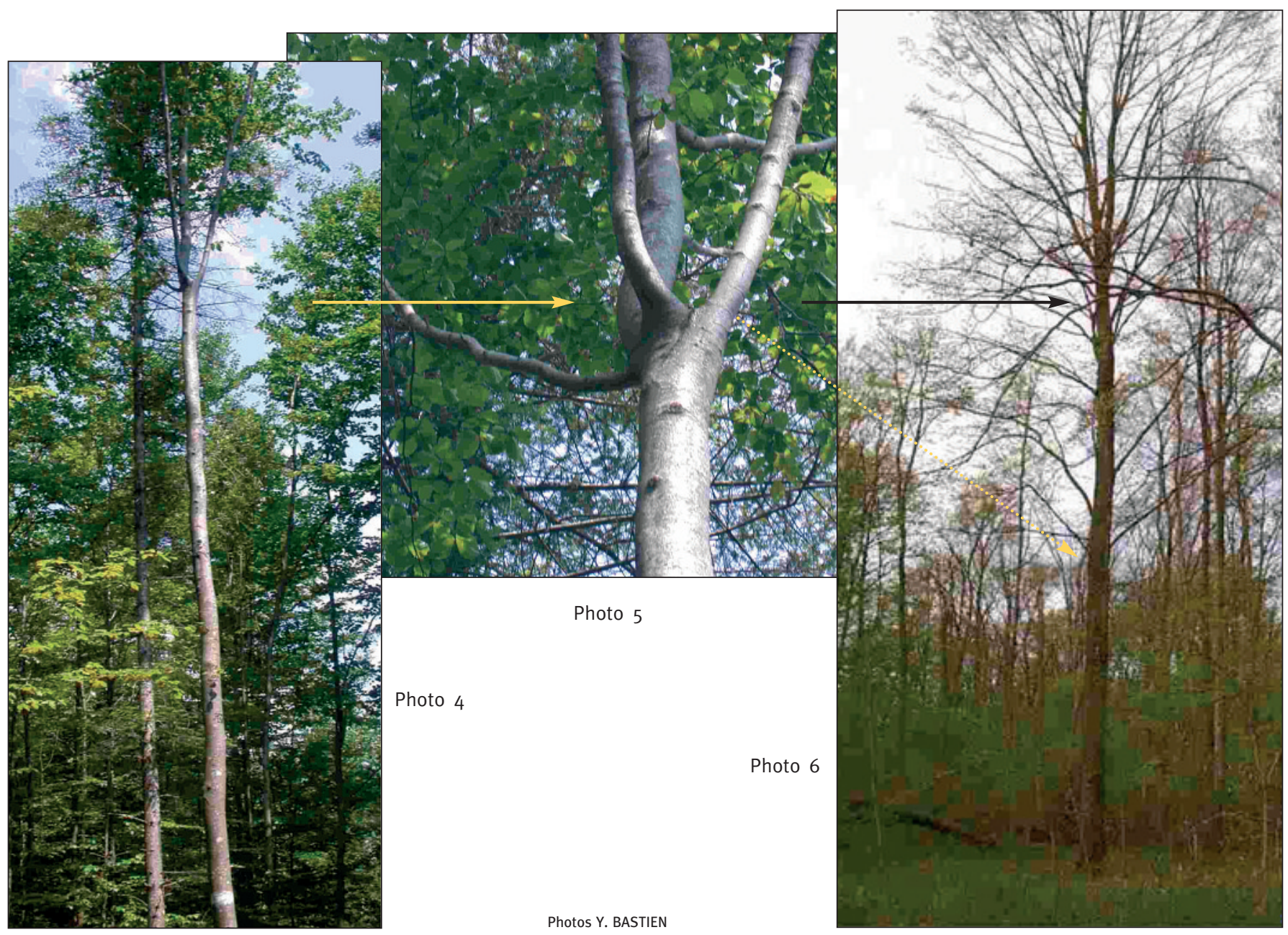

Photo 4 - Branches charpentières bien individualisées à $5 \mathrm{~m}$ de hauteur sur un hêtre-objectif de $13 \mathrm{~m}$ de hauteur totale, fortement éclairci.

Photo 5 - Détail des charpentières constituées de 3 branches dont 2 obliques bien développées en premier plan et d'une branche moins vigoureuse en arrière-plan.

A ce stade, la hauteur de la bille de pied est fixée à ce niveau et les éclaircies devront entretenir en permanence une lumière suffisante pour que la branche la moins vigoureuse puisse grossir ou à défaut se maintenir en vie.

Photo 6 - Gros bois de Hêtre issu de taillis-sous-futaie. La première branche verte (flèche en pointillé) est située à $5 \mathrm{~m}$ de hauteur.

La phase de vieillissement du taillis et de capitalisation de la réserve, durant la période de préparation de la conversion en futaie régulière, a entraîné une remontée du houppier par élagage tardif. On remarque le niveau des branches charpentières à une douzaine de mètres de hauteur. 
Intérêt des faibles matériels sur pied pour maintenir un niveau de mélange suffisant

Les essences de lumière ne se maintiennent dans la hêtraie que si le matériel sur pied reste modéré et en pratiquant des éclaircies par le haut (Pardé, 1981). Ce constat est général dans toutes les futaies régulières de Hêtre denses.

- Une orientation vers la futaie claire à très faible matériel sur pied

II ressort des résultats ci-dessus que l'on s'orientera vers une futaie claire :

- caractérisée par une surface terrière après éclaircie de l'ordre de $15 \mathrm{~m}^{2} /$ ha dans la phase de croissance active (tranche d'âge 30-70 ans) ;

- mélangée d'essences de lumière ${ }^{(3)}$ adaptées aux stations jusqu'au stade mature ;

- à couvert discontinu pour permettre le maintien du mélange ;

- avec des arbres-objectifs à très large houppier et branches charpentières très développées.

- Des éclaircies différenciées selon les stades de développement et des prélèvements adaptés à la forte productivité des hêtraies

Le cycle de croissance de la futaie claire et mélangée de Hêtre se décompose en trois étapes successives (figure 2, p. 118) qui rythmeront les interventions sylvicoles (figure 3, p. 118).

Phase de compression

Elle concerne les semis à bas perchis mélangés en forte concurrence, au sein desquels on dose les essences et on aide les élites à émerger en accompagnant les processus d'évolution naturelle par des interventions limitées au strict minimum (dégagements de semis et nettoiements), (Bastien et Wilhelm, 2000). Cette phase se termine lorsque les fûts des beaux hêtres dominants présentent une hauteur de la première branche verte comprise entre 6 et $8 \mathrm{~m}$ pour une hauteur dominante de 15 à 18 m (Baudran, 1999 ; Demolis, 1991).

Phase de croissance

Elle débute avec les éclaircies ultra fortes par le haut qui peuvent être réalisées en plein ou ponctuellement sous forme de détourage, au profit d'un nombre limité d'arbres-objectifs sélectionnés (50 à 60/ha), dont une majorité de Hêtre et diverses essences économiquement intéressantes en mélange. Le capital sur pied est maintenu à un niveau faible d'environ 15 à $18 \mathrm{~m}^{2}$ de surface terrière à l'ha après éclaircie par des prélèvements très forts (jusqu'à $100 \%$ de l'accroissement courant en volume) pour obtenir une largeur moyenne de cernes de $4 \mathrm{~mm}$ sur les arbres-objectifs orientés vers la production de bois de haute qualité technologique. La rotation des éclaircies est à adapter à l'accroissement courant des peuplements de manière à limiter les prélèvements à un maximum de $100 \mathrm{~m}^{3} / \mathrm{ha}$ à chaque passage. Cette phase se poursuit jusqu'à l'entrée en maturation du peuplement, à partir de 60-70 ans, pour une hauteur dominante du Hêtre comprise entre 25 et $28 \mathrm{~m}$.

Phase de maturation

Elle est caractérisée par un ralentissement de la croissance en hauteur et une concurrence limitée entre les arbres qui présentent un houppier très important dont la base est constituée de grosses branches charpentières. Il est, à ce stade, fondamental de bien gérer la qualité des arbres et d'exclure tout enlèvement de tige de valeur non arrivée à maturité et susceptible de faire baisser

(3) Tous les feuillus précieux, le Chêne sessile, le Pin sylvestre et le Mélèze d’Europe, sont envisageables et nécessitent pour se maintenir dans la hêtraie des éclaircies vigoureuses qui vont dans le sens de l'objectif souhaité. 
Yves Bastien - Sebastian Hein - Alain Chavane

FIGURE 2

LES 3 ÉTAPES DU CYCLE DE CROISSANCE DU HÊTRE

La courbe de croissance de la hauteur dominante $(\mathrm{Ho})$ du Hêtre est une courbe modélisée, Dhôte (1995), d'équation $\mathrm{Ho}=\mathrm{Kp}\left(1-\mathrm{e}^{\text {rcAge }}\right)$ pour la Lorraine pour les meilleures stations (indice de fertilité $\mathrm{Kp}=50$, correspondant au maximum de hauteur atteint par les plus grands arbres), avec un indice rc caractérisant la région climatique. Elle prend en compte les changements de productivité.

Les courbes de Baudran (1999) et Demolis (1991) donnent, pour le Hêtre, 2 modèles d'évolution de la hauteur de la $1^{\text {re }}$ branche verte ( $\mathrm{Hbv}$ ) en fonction de $\mathrm{Ho}$, traduisant ainsi l'évolution de la hauteur potentielle de la bille de pied. Les valeurs indiquées en chiffres sont celles de Hbv données par la courbe de Demolis, très légèrement supérieures à celles de Baudran et qui concernent des peuplements peu éclaircis.

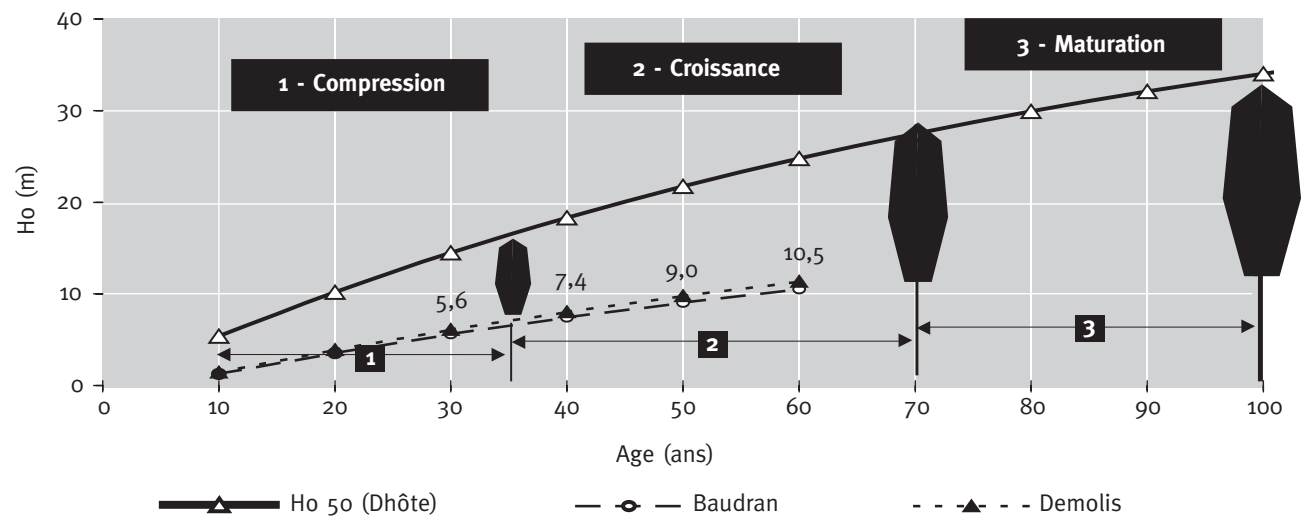

FIGURE 3 GUIDE D’ÉCLAIRCIE EN SURFACE TERRIÈRE (G/HA)

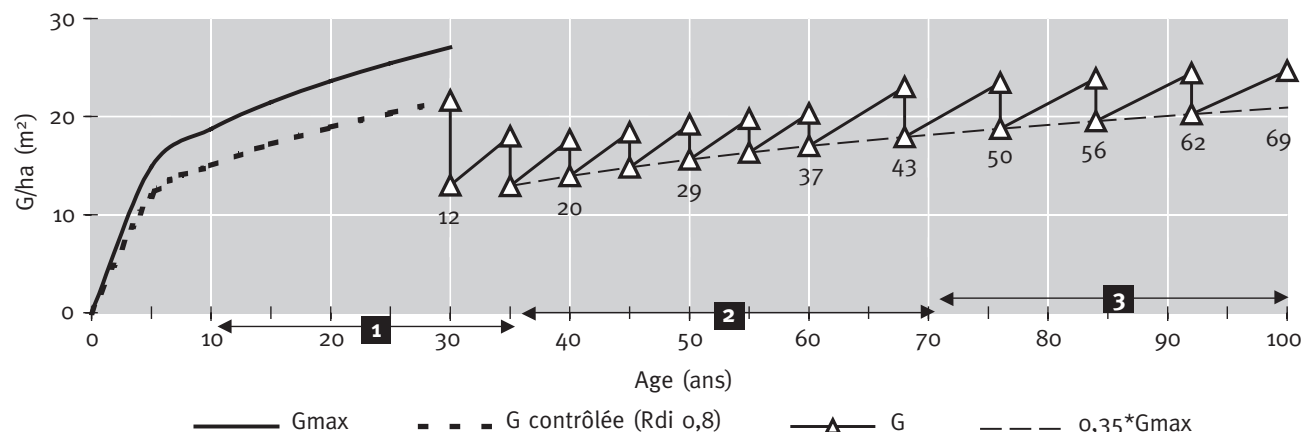

la qualité moyenne du peuplement. Les interventions, dont l'intensité diminue (70 à $80 \%$ de l'accroissement courant en volume), prennent le caractère de coupes sanitaires de récolte progressive d'arbres arrivés au diamètre d'exploitabilité choisi. Les trouées qui en résultent favorisent la forte dynamique du sous-étage d'essences diverses dont certaines tiges vigoureuses et de qualité peuvent avoir vocation à devenir de futurs arbres producteurs.

L'évolution du peuplement est traduite par l'évolution de sa surface terrière à l'hectare (G) en fonction de l'âge pour un niveau de fertilité correspondant à la classe 7 de la table de production pour le Hêtre Nord-Est de Schöber (1972). 
Dans la phase de compression, la courbe Gmax représente l'évolution supposée mais non vérifiée de l'évolution de la surface terrière d'un peuplement en compression évoluant en densité maximale (phénomène d'autoéclaircie) caractérisé par un Rdi (Relative density index) égal à 1 (Dhôte, 1997 ; Le Goff et Ottorini, 1999). Elle n'est sûrement pas valable pour les jeunes peuplements de moins de 30 ans qui sortent du domaine de validité du Rdi.

L'itinéraire sylvicole proposé est défini par la courbe $G$ contrôlée qui traduit une évolution avec des interventions légères en nettoiement, maintenant le peuplement à un niveau théorique de Rdi de o,8. Pour ces deux courbes, très indicatives, de peuplement en phase de compression, les âges sont donnés à titre indicatif dans un intervalle borné de 0-30 ans. Les valeurs de Gmax et de $\mathrm{G}$ contrôlée à 30 ans correspondent aux données que l'on rencontre généralement dans les hêtraies de cet âge, avant la première éclaircie.

Pour les phases de croissance (30-70 ans) et de maturation (70-100 ans), le scénario d'éclaircie courbe G (donnée avant et après éclaircie) est basé sur la simulation suivante :

- croissance en diamètre fixée telle que la largeur de cerne moyenne annuelle soit de $2 \mathrm{~mm}$ pendant la phase de compression (0-30 ans) et de $4 \mathrm{~mm}$ ensuite (les diamètres figurent en chiffres grisés en bas de la courbe $\left.0,35^{\star} \mathrm{Gmax}\right)$;

- accroissement en surface terrière égal à $100 \%$ de celui donné par la classe 7 de Schöber ;

- rotations de 5 ans de 30 à 60 ans puis 8 ans au-delà ;

- prélèvements calculés de manière à ramener le peuplement à un Rdi de 0,35 et donc à une surface terrière après éclaircie $(\mathrm{G})$ de $35 \%$ de $\operatorname{Gmax}(0,35$ Gmax).

Cette sylviculture, qui rompt avec le traitement classique de futaie régulière du Hêtre, s'appuie sur des indicateurs biologiques qui permettront de suivre l'évolution dans le temps de l'écosystème hêtraie caractérisé par :

- un sous-étage complet d'allure jardinée d'essences tolérant l'ombrage : Hêtre, Sapin, Charme, Érables ;

- un niveau de mélange suffisant d'essences de lumière dans le peuplement principal, au début de la phase de maturation (20\% minimum en surface terrière) ;

- des arbres-objectifs à grosses branches charpentières vigoureuses constituant la base élargie d'un important houppier vivant, représentant pour l'arbre mûr au moins $50 \%$ de sa hauteur totale afin d'obtenir une bille de 6 à $10 \mathrm{~m}$ de hauteur de pied et d'un diamètre minimum de $60 \mathrm{~cm}$ à $1,30 \mathrm{~m}$ de hauteur en moins de 100 ans.

\section{En futaie irrégulière}

La formalisation de la sylviculture du Hêtre en futaie irrégulière est en cours mais des résultats sont déjà mesurables en de nombreux points tant cette essence se régénère bien sous couvert.

Le travail actuel, mené par l'Association pour la Futaie irrégulière (AFI) et basé sur l'étude de l'évolution de peuplements dans différents contextes écologiques, apporte un certain nombre de résultats intéressants pour les peuplements à base de Hêtre issus d'anciens taillis-sous-futaie. Pour résumer, on peut retenir que l'évolution progressive vers la futaie irrégulière est commandée par un chiffre-clé caractérisant bien le niveau de compétition à l'aide de la surface terrière des peuplements à un total de l'ordre de $15-18 \mathrm{~m}^{2} / \mathrm{ha}$ après coupe, réparti entre les arbres précomptables (diamètre supérieur ou égal à $20 \mathrm{~cm}$ ) et les brins (futaie ou taillis de diamètre inférieur à $20 \mathrm{~cm})$.

Il s'agit d'un seuil maximum à raisonner de manière combinée et qui demande à être nuancé en fonction des potentialités des stations forestières et des essences en mélange. 
Yves Bastien - Sebastian Hein - Alain Chavane

L'exemple de la hêtraie d'Alain Chavane dans les Vosges (région de Darney) illustre l'évolution intéressante d'une hêtraie acidiphile mélangée de Chêne sessile et Sapin, à partir d'un ancien taillis-sous-futaie enrichi (figure 4 , ci-dessous).

\section{FIGURE 4 ÉVOLUTION DE LA SURFACE TERRIÈRE D'UNE HÊTRAIE IRRÉGULIÈRE PRIVÉE, GÉRÉE PAR ALAIN CHAVANE}

(la Quiquengrogne, région de Darney, Vosges)

Sources : Alain Chavane et AFI (Association pour la Futaie irrégulière)

Hêtraie à Chêne sessile et Sapin

Gestion

- Taillis-sous-futaie jusqu'en 1945 à la révolution de 25 ans.

- 1945-1975 : enrichissement, arrêt des coupes de taillis, progression du Sapin introduit par Broilliard (vers 1870).

- À partir de 1975, gestion en futaie irrégulière par coupes fortes à la rotation de 12 ans (gestion Alain Chavane).

- 3 balivages intensifs au cours des années $1985 / 2000$.

- Récolte des gros hêtres en 1982 et 1993, et des sapins en 1990.

- Peuplement actuel

Futaie irrégulière à bois moyens avec régénération par bouquets de Hêtre et Sapin.

Composition (essences en \% de G) : Hêtre $81 \%$, Chêne sessile 14 , Sapin $5 \%$. Forte dynamique du Sapin et du Hêtre.

Hêtre de bonne qualité ( $51 \%$ de G Hêtre de qualité A et B).

Production courante : $9 \mathrm{~m}^{3} / \mathrm{ha} / \mathrm{an}$.

- Sylviculture actuelle

Culture d'arbres en croissance libre favorisant les arbres de qualité, le mélange des essences et l'irrégularité des peuplements.

Récolte progressive au diamètre d'exploitabilité avec coupe à rotation abaissée à 8 ans dans le futur plan simple de gestion.

\section{CONCLUSIONS}

Au niveau de la sylviculture du Hêtre, on peut dégager deux grands axes complémentaires de stratégie :

- augmenter la qualité de la production ;

- conduire les peuplements à un faible niveau de matériel sur pied.

De ces deux axes découle naturellement, de par la dynamique du mélange des essences et de la régénération naturelle continue, l'obtention d'un écosystème hêtraie plus résilient et à moindres risques eu égard à divers facteurs défavorables (tempête, changement climatique).

Dans les choix sylvicoles d'aujourd'hui qui façonnent la hêtraie de demain, il convient de dépasser le cadre habituel de gestion et d'être très attentif à l'évolution des peuplements, tant sur la dynamique de croissance que sur la qualité des biens et des services produits - la qualité des 
arbres jouant bien entendu un rôle important - . Traitée en futaie régulière ou irrégulière, la hêtraie mélangée conduite en futaie claire aura sans doute une évolution très différente de celle des peuplements actuels. Dans cette vision dynamique très ouverte, on peut penser à différentes options de gestion possibles. Au forestier d'aujourd'hui de s'y préparer !
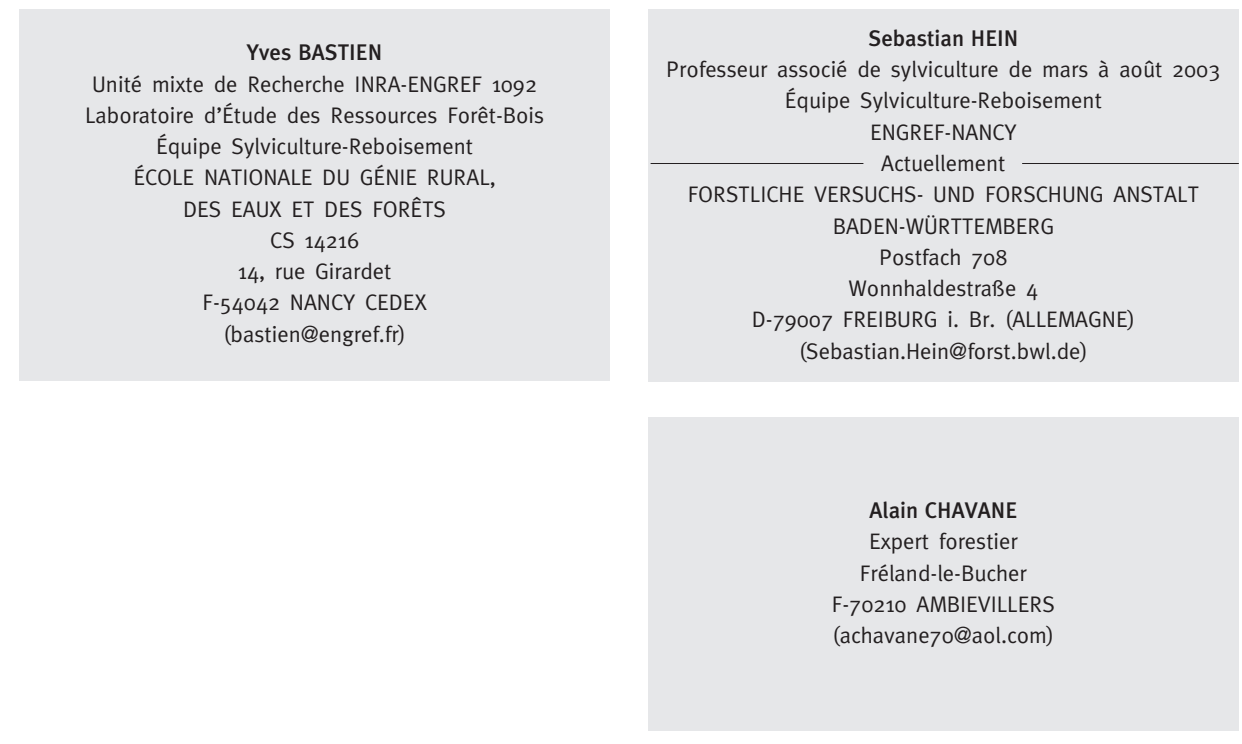

\section{BIBLIOGRAPHIE}

AFNOR. - Norme européenne de classement des bois ronds feuillus. Partie 1 : Chêne et Hêtre. - Association française de Normalisation, 1997.

ARNAL (M.), DROUINEAU (S.). - Diagnostic et propositions de normes sylvicoles de rattrapage des peuplements réguliers de Hêtre et Chêne sessile en Normandie. - Nancy : ENGREF, 1997. - 113 p. + annexes (Mémoire de stage de $3^{\mathrm{e}}$ année FIF).

BASTIEN (Y.). - Pour l’éducation du Hêtre en futaie claire et mélangée. - Revue forestière française, vol. XLIX, $\mathrm{n}^{\circ} 1$, 1997, pp. 49-68.

BASTIEN (Y.), OTTO (H.J.). - La Théorie des groupes d'arbres : application aux éclaircies de futaie régulière. - Revue forestière française, vol. $\mathrm{L}, \mathrm{n}^{\circ}$ 3, 1998, pp. 251-262.

BASTIEN (Y.), WILHELM (G.). - Une sylviculture d'arbres pour produire des gros bois de qualité. - Revue forestière française, vol. LII, $\mathrm{n}^{\circ} 5,2000$, pp. 407-424.

BAUDRAN (C.). - Modélisation de la hauteur de la base du houppier chez le Hêtre (Fagus sylvatica L.) à partir de données dendrométriques de 21 peuplements lorrains. - Université de Nancy I, 1999. - 17 p. (Rapport DEA de Biologie forestière).

BONTEMPS (J.-D.). - Évolution de la vitesse de croissance radiale des hêtraies. - Communication présentée au séminaire du LERFOB - ENGREF Nancy du 9 janvier 2003.

COMTESSE (E.). - Propositions d'itinéraires sylvicoles pour les futaies régulières de Hêtre en Picardie. Nancy : ENGREF, 2000. - 46 p. + annexes (Mémoire de stage de $3^{\mathrm{e}}$ année FIF).

DEMOLIS (C.). - Influence de la sylviculture sur l'élagage du Hêtre. - Bulletin technique de l'Office national des Forêts, n० 21, 1991, pp. 31-42.

DHÔTE (J.-F.). - Définition de scénarios d'éclaircie pour le Hêtre et le Chêne. - Revue forestière française, vol. XLVII, $\mathrm{n}^{\circ}$ spécial “Modélisation de la croissance des arbres forestiers et de la qualité des bois”, 1995, pp. 106-110. 
Yves Bastien - Sebastian Hein - Alain Chavane

DHÔTE (J.-F.). - Effet des éclaircies sur le diamètre dominant dans des futaies de Hêtre et de Chêne. Revue forestière française, vol. XLIX, nº 6, 1997, pp. 557-578.

DHÔTE (J.-F.), DUPOUEY (J.-L.), BERGÈS (L.). - Modifications à long terme déjà constatées de la productivité des forêts françaises. - Revue forestière française, vol. LII, $\mathrm{n}^{\circ}$ spécial "Conséquences des changements climatiques pour la forêt et la sylviculture”, 2000, pp. 37-48.

LE GOFF (N.), OTTORINI (J.-M.). - Effets des éclaircies sur la croissance du Hêtre. Interaction avec les facteurs climatiques. - Revue forestière française, vol. LI, $\mathrm{n}^{\circ}$ 2, 1999, pp. 355-364.

PARDÉ (J.). - De 1882 à 1976/80, les places d'expérience de sylviculture du Hêtre en forêt domaniale de Haye (Meurthe-et-Moselle). - Revue forestière française, vol. XXXIII, nº spécial "Sylvicultures en futaies feuillues", 1981, pp. 41-64.

SEYNAVE (I.). - Propositions de scénarios sylvicoles pour les futaies régulières de Hêtre en Lorraine. Rapport technique ONF Lorraine, 1999. - 29 p. + annexes.

VINKLER (I.). - Effets des caractéristiques dendrométriques des hêtraies du quart Nord-Est sur le niveau des dégâts. In : Séminaire "Forêt, Vent et risques". - Paris : GIP ECOFOR, 2002.

\section{SYLUICULTURE DU hÊTRE : CONTRAINTES, ENJEUX, ORIENTATIONS DE GESTION [Résumé]}

Occupant une surface totale de près de 1300 ooo ha, le Hêtre représente en France un enjeu économique important. Les futaies régulières de Hêtre, qui en constituent une part majoritaire, sont caractérisées par des peuplements denses et purs, sensibles aux dégâts de vent, et en forte augmentation de productivité. Les peuplements de taillis-sous-futaie qui se révèlent plus diversifiés, sont orientés dans différents schémas de conversion en futaie.

Les enjeux de gestion actuelle visent à mieux gérer les risques et à améliorer la production qualitative en limitant les coûts. Pour la futaie régulière, les orientations qui en découlent reposent sur une très forte intensification des éclaircies dans les peuplements en croissance (fourchette d'âge comprise entre 30 et 70 ans) en contenant leur accroissement courant afin de maintenir une surface terrière après éclaircie à $15-18 \mathrm{~m}^{2} / \mathrm{ha}$ et de favoriser le mélange d'essences de lumière. Après 70 ans, les éclaircies prennent le caractère de coupes sanitaires de récolte. Pour les peuplements hérités du taillis-sous-futaie à forte variabilité structurelle, leur évolution progressive vers la futaie irrégulière passe par leur éducation à un faible niveau de capital sur pied.

L'évolution des traitements de futaie régulière et irrégulière de Hêtre converge vers une culture de peuplements à faible matériel sur pied. Cette caractéristique, en favorisant le mélange d'essences et l'obtention de bois à larges cernes, améliore la résilience des peuplements et la qualité de leur production.

\section{BEECH SILUICULTUAE - CONSTRAINTS, ImPLICATIONS, MANAGEMENT GUIDELINES [Rbstract]}

Beech, which covers a surface area of nearly 1300000 ha, is a considerable commercial interest in France. Regular high beech forest, that accounts for the major portion of that area, is made up of dense, singlespecies stands that are sensitive to wind damage and increasingly productive. The coppice-with-standards stands are more mixed and managed under various schemes for conversion to high forest.

The focus of current management is to better provide for risks and enhance quality while keeping costs down. Guidelines for regular high forest are based on highly intensive thinning in growing stands (aged between 30 and 70 years) and limiting their current increment so as to maintain a basal area after thinning of $15-18 \mathrm{~m}^{2} / \mathrm{ha}$ and encourage mixtures with shade-intolerant species. After the age of 70 , thinning is performed as sanitation felling. For stands grown under a coppice-with-standards system that are structurally highly diverse, gradual conversion to irregular high forest requires education at a low level of capital stock.

Approaches to even and uneven high forest treatment for beech are changing and tending towards stand cultivation with low stocking levels that fosters mixtures of species and produces broad growth rings thereby enhancing stand resilience and product quality. 